\title{
One-step, facile synthesis of pyrazolopyridines and tetrahydropyrazolopyridines through disproportionation of initially formed pyrazolo Hantzsch dihydropyridine
}

\author{
Chuanxiang Liu, Zhong Li,* Liwei Zhao, and Li Shen
}

Shanghai Key Lab of Chemical Biology; School of Pharmacy, East China University of Science and Technology, P.O. Box 544 Shanghai 200237, PR. China

E-mail: lizhong@ecust.edu.cn

\begin{abstract}
We have developed a single-step synthesis of pyrazolopyridines and hydropyrazolopyridines by condensation 3-carbonitrile-5-aminopyrazole (1) with seven substituted $\alpha, \beta$-unsaturated aldehydes in acid medium. Straightforward access to various pyrazolopyridines and hydropyrazolopyridines was achieved through disproportionation of initially formed pyrazolo Hantzsch dihydropyridines. All the pyrazolopyridines and hydropyrazolopyridines were well characterized by spectroscopic analyses.
\end{abstract}

Keywords: Pyrazolopyridine, heterocycles , Hantzsch, disproportionation

\section{Introduction}

Pyrazolopyridines and its hydroderivatives are very interesting pyrazole derivatives with wideranging biological activities. ${ }^{1}$ A number of pyrazolo[3,4- $\left.b\right]$ pyridines exhibit a wide range of biological activities, including interesting anxiolytic activity (e.g. tracazolate), dopamine D3 receptor antagonist, antiherpetic and antiallergic properties. ${ }^{2}$ Usually, pyrazolopyridines can be synthesized via (i) 1,3-dipolar cycloaddition reaction from azomethine imines and alkynes; (ii) cyclocondensations of aminoazoles and aminoazines with $\alpha, \beta$-unsaturated aldehydes and ketones containing at least two active hydrogen atoms. ${ }^{3}$ While considerable progress has been made in synthesis of pyrazolo[3,4- $b$ ]pyridines, its hydroderivatives ${ }^{4}$ such as 4,5,6,7-tetrahydropyrazolopyridines and 4,7-dihydropyrazolopyridines has received much less attention comparatively.

The rapid assembly of molecular diversity is a significant goal of synthetic organic chemistry and one of the key paradigms of modern drug discovery. Great interest has been accumulated in recent years in the chemistry of Hantzsch 1,4-dihydropyridines upon many striking discoveries of bioactive roles ${ }^{5}$ as potent blockers of calcium $\left(\mathrm{Ca}^{2+}\right)$ currents, and application in synthetic and physical organic chemistry as attractive biomimetic reducing agents. ${ }^{6}$ Recently some reports about Hantzsch 1,4-dihydropyridines as external reductant have shown attractive application in 
hydrogenations of olefins, ${ }^{7}$ asymmetric reductions, ${ }^{8}$ reductive aminations, ${ }^{9}$ reduction of $\alpha, \beta$ epoxyketones. ${ }^{10} \mathrm{~A}$ common approach ${ }^{11}$ involving disproportionation reaction of initially formed 1,4-dihydropyridines can be explained for synthesis of tetrahydropyridines. To our best knowledge, there is no report about disproportionation of initially formed unstable heterocyclic-fused Hantzsch dihydropyridines, such as pyrazolo Hantzsch dihydropyridines. Therefore, we reasoned that it might be applicable to facilitate development of synthesis of pyrazolopyridines and tetrahydropyrazolopyridines for potential modern drug discovery. (Figure 1)

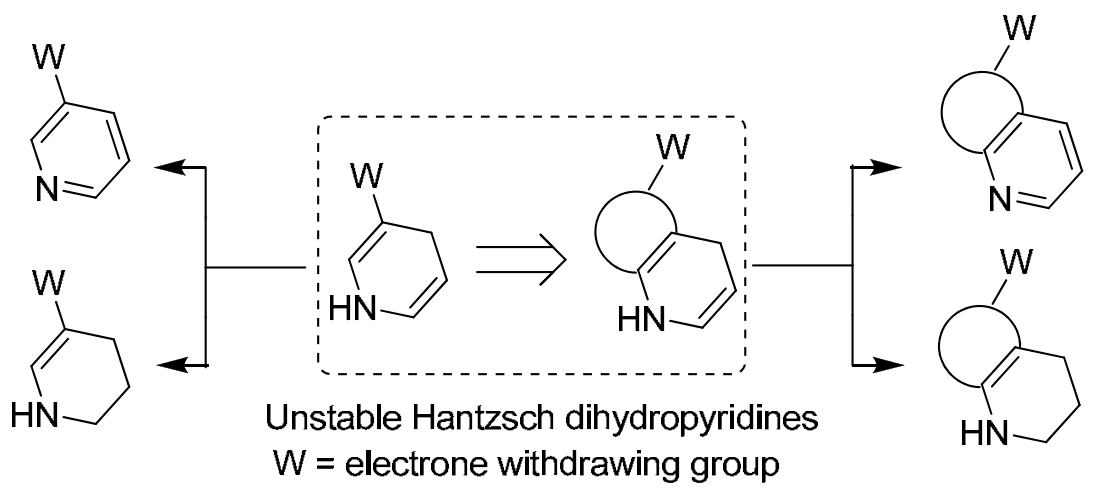

\section{Figure 1}

3 -(Methyl or phenyl)-5-aminopyrazoles, have been reported ${ }^{12}$ as the substance to react with $\alpha, \beta$ unsaturated aldehydes and ketones containing at least two active hydrogen atoms. To our best knowledge, there is no report about strong electron-withdrawing substitute such as $\mathrm{CN}$ group in pyrazole 3-position as the substance to research the synthetic pathways to fused pyrazolopyridines. 5-amino-1-(2,6-dichloro-4-(trifluoromethyl)-phenyl)-1H-pyrazole-3-carbonitrile (1), which is a key intermediate in synthesis of Fipronil ${ }^{13}$, have three nucleophilic centers including its $\alpha$-carbon atom and amino groups to react with electrophiles. In the present work, we describe that one-step synthesis of pyrazolopyridines and tetrahydropyrazolopyridines by condensation compound $\mathbf{1}$ with substituted $\alpha, \beta$-unsaturated aldehydes in acid medium (Scheme 1).

\section{Results and Discussion}

In case of acetic acid as catalyst, the common Schiff bases 8-13 were synthesized in good yields. But for hydrochloric acid, two kinds of the products including pyrazolopyridines and tetrahydropyrazolopyridines were synthesized in moderate yields (Scheme 1 and Table 1). The most obvious products are pyrazolopyridine (19-24), and another is the formation of tetrahydropyrazolopyridine (14-18) in minor yields. For 7 as the reactant in hydrochloric acid, formation of 4,7-dihydro-pyrazolopyridine (25) through a further molecular condensation was investigated firstly. 


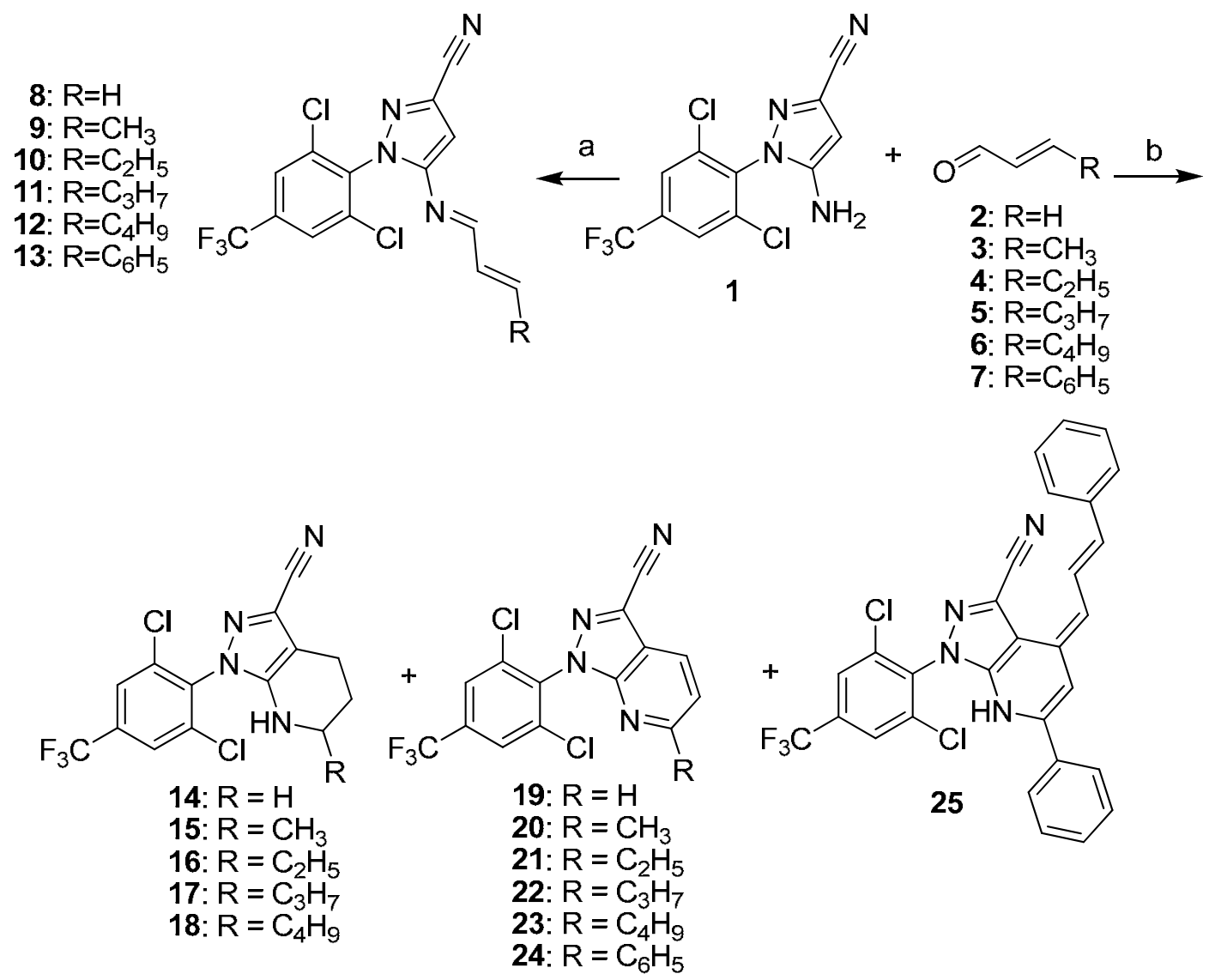

Scheme 1. Reagents and reaction conditions: (a) $\mathrm{AcOH}, \mathrm{CH}_{3} \mathrm{CN}, 50{ }^{\circ} \mathrm{C}$. (b) conc- $\mathrm{HCl}, \mathrm{CH}_{3} \mathrm{CN}, 50$ ${ }^{\circ} \mathrm{C}$.

Table 1. The obtained products (Yield ${ }^{\mathrm{a}}$ ) in different acid catalysis

\begin{tabular}{cccc}
\hline $\mathrm{RCH}=\mathrm{CHCHO}$ & \multicolumn{2}{c}{ Cat. $\mathrm{HCl},(\%)$} & Cat. $\mathrm{AcOH},(\%)$ \\
\hline $\mathrm{H}^{\mathrm{b}}$ & $\mathbf{1 4}(30 \%)$ & $\mathbf{1 9}(58 \%)$ & $\mathbf{8}(82 \%)$ \\
$\mathrm{CH}_{3}$ & $\mathbf{1 5}(24 \%)$ & $\mathbf{2 0}(50 \%)$ & $\mathbf{9}(80 \%)$ \\
$\mathrm{C}_{2} \mathrm{H}_{5}$ & $\mathbf{1 6}(17 \%)$ & $\mathbf{2 1}(48 \%)$ & $\mathbf{1 0}(75 \%)$ \\
$\mathrm{C}_{3} \mathrm{H}_{7}$ & $\mathbf{1 7}(18 \%)$ & $\mathbf{2 2}(45 \%)$ & $\mathbf{1 1}(70 \%)$ \\
$\mathrm{C}_{4} \mathrm{H}_{9}$ & $\mathbf{1 8}(15 \%)$ & $\mathbf{2 3}(43 \%)$ & $\mathbf{1 2}(70 \%)$ \\
$\mathrm{C}_{6} \mathrm{H}_{5}$ & $\mathbf{2 5}(15 \%)$ & $\mathbf{2 4}(40 \%)$ & $\mathbf{1 3}(75 \%)$ \\
\hline
\end{tabular}

${ }^{a}$ Isolated yield. ${ }^{b}$ Determined by ${ }^{1} \mathrm{H}$ NMR of crude reaction mixtures (supporting information).

The mechanism for the formation of pyrazolo[3,4-b]pyridines and tetrahydropyrazolo $[3,4-$ $b$ ]pyridines is depicted in Scheme 2. In the initial step, the Michael adduct was formed, which could undergo dehydration reaction to intermediate $\mathbf{B}$. There are probably two pathways for intermediate B, such as aromatization in the oxygen by loosing water to obtain the corresponding products (19- 
24) (Skraup reaction ${ }^{14}$ ) and further tautomerism to form pyrazolo Hantzsch dihydropyridines $\mathbf{A}$. The calculated energy of this unstable Hantzsch analog $\mathbf{A}$ is $37.20 \mathrm{kcal} / \mathrm{mol}$, which is unstable than the intermediate $\mathbf{B}(27.66 \mathrm{kcal} / \mathrm{mol})$. And also, disproportionation of initially formed pyrazolo Hantzsch dihydropyridines A can provide the corresponding products (14-18, 19-24). The mainly products (19-24) was ascribed to the two potential different reactive routes above.<smiles></smiles>

1<smiles>[R]C1CC(O)c2c(C#N)nn(-c3c(Cl)cc(C(F)(F)F)cc3Cl)c2N1</smiles>

Michael addition<smiles>[R]C1=CCc2c(C#N)nn(-c3c(Cl)cc(C(F)(F)F)cc3Cl)c2N1</smiles>

[pyrazolo Hantzsch dihydropyridine]<smiles>[Y]CC(C)C([R5])[O-]</smiles>

14: $\mathrm{R}=\mathrm{H}$

15: $\mathrm{R}=\mathrm{CH}_{3}$

16: $\mathrm{R}=\mathrm{C}_{2} \mathrm{H}_{5}$

17: $\mathrm{R}=\mathrm{C}_{3} \mathrm{H}_{7}$

18: $\mathrm{R}=\mathrm{C}_{4} \mathrm{H}_{9}$<smiles>[R]C1C=Cc2c(C#N)nn(-c3c(Cl)cc(C(F)(F)F)cc3Cl)c2N1</smiles>

B
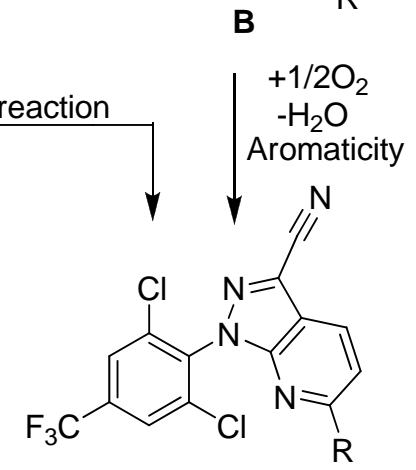

19: $\mathrm{R}=\mathrm{H}$

20: $\mathrm{R}=\mathrm{CH}_{3}$

21: $\mathrm{R}=\mathrm{C}_{2} \mathrm{H}_{5}$

22: $\mathrm{R}=\mathrm{C}_{3} \mathrm{H}_{7}$

23: $\mathrm{R}=\mathrm{C}_{4} \mathrm{H}_{9}$

24: $\mathrm{R}=\mathrm{C}_{6} \mathrm{H}_{5}$
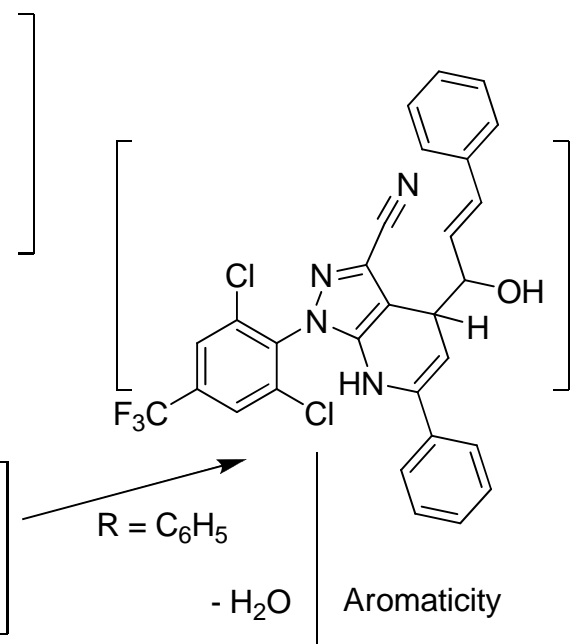

Scheme 2. Proposed reaction mechanism and stepwise formation of the final products.

For 7, further molecular condensation between $\mathbf{B}$ with excess 7, rearrangement and then dehydration to obtain the investigated 25 . The final 4,7 -dihydro- pyrazolopyridine $\left(25^{15}\right)$ is more stable compared to 4,5,6,7-tetrahydro-pyrazolopyridine in cinnamaldehyde case due to the formation of conjugated $\pi$-electron systems.

Further exploring the reaction of 1 with $(E)$-2-methylpent-2-enal (26), it was found that the product 27 was obtained in high yield (70\%). The corresponding hydropyrazolopyridine was not found, which indicate that the pyrazol Hantzsch dihydropyridine may be not formed. This can be 
explained by that the steric effect of $\alpha$-methyl (26) inhibited the tautomerism to form the unstable pyrazolo Hantzsch dihydropyridine. (Scheme 3)

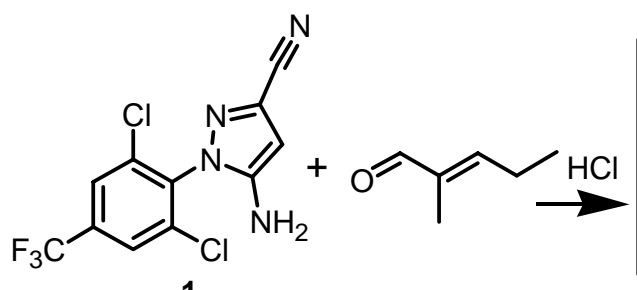

1<smiles>CCC1Nc2c(c(C#N)nn2-c2c(Cl)cc(C(F)(F)F)cc2Cl)C1O</smiles>

Michael addition<smiles>Cc1cc(N)n(-c2c(Cl)cc(C(F)(F)F)cc2Cl)n1</smiles>
28(yeild 70\%)

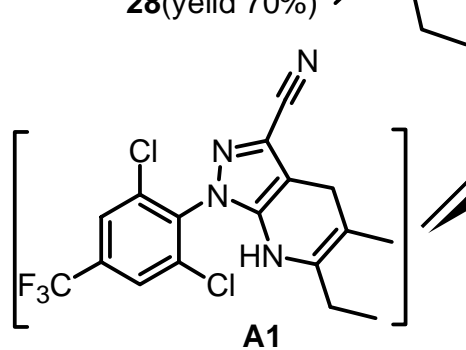

A1

[pyrazolo Hantzsch dihydropyridine]

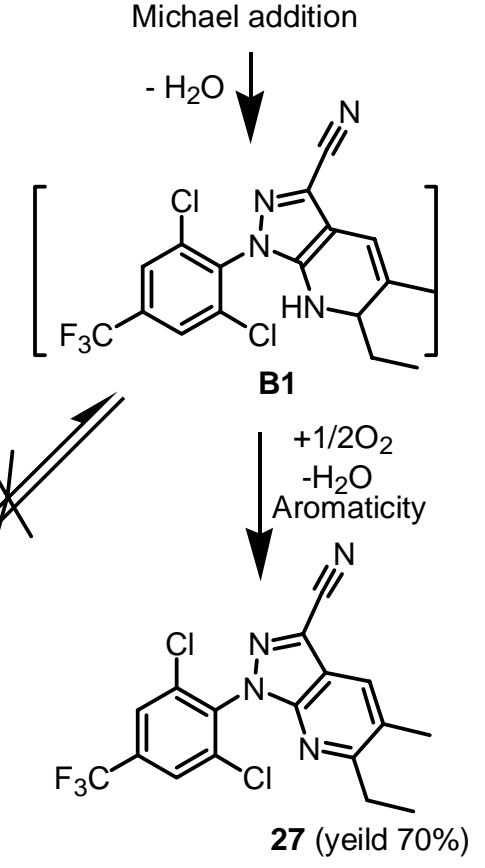

Scheme 3. Proposed reaction mechanism of 1 with 26.

\section{Conclusions}

In conclusion, we have demonstrated one-step reaction of 3-carbonitrile-5-aminopyrazole (1) with substituted $\alpha, \beta$-unsaturated aldehyde $(\mathbf{2 - 7}, \mathbf{2 6})$, which results in the formation of substituted pyrazolopyridines and tetrahydropyrazolopyridines through disproportionation of initially formed pyrazolo Hantzsch dihydropyridines. Further investigations on unstable Hantzsch analog for rapid assembly of molecular diversity may expand more exquisite heterocyclic compounds possessing potential bioactivities for modern drug discovery.

\section{Experimental Section}

General Procedures. Experiments were performed under a dry nitrogen atmosphere. Melting points were taken on a micro melting point apparatus made in Beijing and were uncorrected. ${ }^{1} \mathrm{H}$ NMR (400 MHz) and ${ }^{13} \mathrm{C}$ NMR $(100 \mathrm{MHz})$ spectra were recorded on a Bruker AVANCE $400 \mathrm{MHz}$ 
spectrometer using $\mathrm{CDCl}_{3}$ as solvent at $298 \mathrm{~K}$ and TMS as an internal standard. IR spectra were recorded on a Nicolet Magna-IR 550 instrument using KBr pellets. High Resolution Mass spectra were obtained on MicroMass GCT CA 055 spectrometers. Analytical thin-layer chromatography (TLC) was carried out on precoated plated (silica gel $60_{\mathrm{F} 254}$ ), and spots were visualized with ultraviolet light. All chemicals or reagents were purchased from standard commercial suppliers.

Hydropyrazolopyridines(14-18, 25), pyrazolopyridines(19-24, 27) and the common Schiff bases (8-13, 28). Preparation of target compounds; typical procedure

A flame-dried flask was charged with 1 (640 mg, $2 \mathrm{mmol}), \alpha, \beta$-unsaturated aldehyde (1.5 mmol) and $\mathrm{CH}_{3} \mathrm{CN}(10 \mathrm{~mL})$. Then a catalytic amount $\left(3\right.$ drops) of $\mathrm{CH}_{3} \mathrm{COOH}$ or $\mathrm{HCl}$ was added. The reaction mixture was heated at $50{ }^{\circ} \mathrm{C}$ for $6-24 \mathrm{~h}$. Water $(10 \mathrm{~mL})$ was added and extracted with $\mathrm{CH}_{2} \mathrm{Cl}_{2}(3 \times 10 \mathrm{~mL})$. The combined organic phase was washed with brine and dried over anhydrous $\mathrm{Na}_{2} \mathrm{SO}_{4}$. The organic layer was separated and dried over anhydrous sodium sulfate, and the methylene chloride was removed under reduced pressure. The crude mixture was purified on a silica gel column chromatography using the hexane/EA as solvent to give target compounds.

(E)-5-(Allylideneamino)-1-(2,6-dichloro-4-(trifluoromethyl)phenyl)-1H-pyrazole-3-

carbonitrile (8). $\mathrm{Mp} 99.5-101.3{ }^{\circ} \mathrm{C}$. IR (KBr): 3188, 2239, 1688, 1320, 1182, 783, $675 \mathrm{~cm}^{-1} .{ }^{1} \mathrm{H}$ $\operatorname{NMR}\left(400 \mathrm{MHz}, \mathrm{CDCl}_{3}, 25^{\circ} \mathrm{C}\right): \delta=6.03-6.13\left(\mathrm{~m}, 2 \mathrm{H}, \mathrm{CH}_{2}\right), 6.50-6.54(\mathrm{~m}, 1 \mathrm{H}, \mathrm{CH}=\mathrm{CH}), 6.69(\mathrm{~s}$, $1 \mathrm{H}$, Pyrazole-H), 7.74 (s, $2 \mathrm{H}, \mathrm{ArH}), 8.30$ (d, $J=9.1 \mathrm{~Hz}, 1 \mathrm{H}, \mathrm{N}=\mathrm{CH}) .{ }^{13} \mathrm{C} \mathrm{NMR}\left(100 \mathrm{MHz}, \mathrm{CDCl}_{3}\right.$, $\left.25{ }^{\circ} \mathrm{C}\right): \delta=166.1,152.3,142.7,136.8,136.0,133.9,127.6,126.2,125.7,122.1,113.3,97.1$. EIMS: $m / z(\%)=358[\mathrm{M}]^{+}$(27.61), 323 (34.44), 287 (16.91), 273 (20.50), 271 (100.00), 236 (14.66), 215 (22.12), 213 (40.06), 178 (12.73), 143 (12.08), 66 (15.03), 39 (32.55). HRMass(EI): m/z Calcd for $\mathrm{C}_{14} \mathrm{H}_{7} \mathrm{Cl}_{2} \mathrm{~F}_{3} \mathrm{~N}_{4}$ : 358.0000; found: 358.0004 .

5-((E)-((E)-But-2-enylidene)amino)-1-(2,6-dichloro-4-(trifluoromethyl)phenyl)-1H-pyrazole-3-

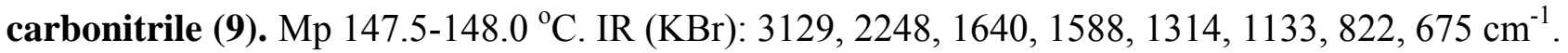
${ }^{1} \mathrm{H}$ NMR $\left(400 \mathrm{MHz}, \mathrm{CDCl}_{3}, 25{ }^{\circ} \mathrm{C}\right): \delta=1.98\left(\mathrm{~d}, J=6.8 \mathrm{~Hz}, 3 \mathrm{H}, \mathrm{CH}_{3}\right), 6.30\left(\mathrm{dd}, J_{1}=7.0 \mathrm{~Hz}, J_{2}=\right.$ $8.2 \mathrm{~Hz}, 1 \mathrm{H}, \mathrm{CH}=\mathrm{CH}), 6.67-6.58$ (m, $2 \mathrm{H}, \mathrm{CH}$, Pyrazole-H), 7.73 (s, $2 \mathrm{H}, \mathrm{ArH}), 8.24$ (d, J=9.2 Hz, $1 \mathrm{H}, \mathrm{N}=\mathrm{CH}) .{ }^{13} \mathrm{C}$ NMR $\left(100 \mathrm{MHz}, \mathrm{CDCl}_{3}, 25{ }^{\circ} \mathrm{C}\right): \delta=166.2,153.0,149.2,137.0,136.0,133.8$, 132.1, 127.5, 126.0, 122.2, 113.4, 96.7, 19.1. EIMS: $m / z(\%)=372[\mathrm{M}]^{+}(62.79), 337(100.00), 287$ (23.77), 285 (33.91), 283 (4.12), 270 (7.74), 250 (6.54), 213 (24.22), 178 (7.71), 80 (11.94), 53 (28.62). HRMass(EI): $\mathrm{m} / z$ Calcd for $\mathrm{C}_{15} \mathrm{H}_{9} \mathrm{Cl}_{2} \mathrm{~F}_{3} \mathrm{~N}_{4}$ : 372.0156; found: 372.0153 .

1-(2,6-Dichloro-4-(trifluoromethyl)phenyl)-5-((E)-((E)-pent-2-enylidene)amino)-1H-pyrazole-

3-carbonitrile (10). $\mathrm{Mp} 124.9$ - $125.4^{\circ} \mathrm{C}$. IR (KBr): 3130, 2247, 1646, 1587, 1310, 1130, 820, 675 $\mathrm{cm}^{-1} .{ }^{1} \mathrm{H}$ NMR $\left(400 \mathrm{MHz}, \mathrm{CDCl}_{3}, 25^{\circ} \mathrm{C}\right): \delta=1.10\left(\mathrm{t}, J=7.2 \mathrm{~Hz}, 3 \mathrm{H}, \mathrm{CH}_{3}\right), 2.30-2.35(\mathrm{~m}, 2 \mathrm{H}$, $\left.\mathrm{CH}_{2}\right)$, 6.25-6.31 (m, $\left.1 \mathrm{H}, \mathrm{CH}=\mathrm{CH}\right), 6.62$ (s, $1 \mathrm{H}$, Pyrazole-H), 6.63-6.71 (m, $\left.1 \mathrm{H}, \mathrm{CH}\right), 7.74(\mathrm{~s}, 2 \mathrm{H}$, $\operatorname{ArH}), 8.26(\mathrm{~d}, J=9.2 \mathrm{~Hz}, 1 \mathrm{H}, \mathrm{N}=\mathrm{CH}) .{ }^{13} \mathrm{C} \mathrm{NMR}\left(100 \mathrm{MHz}, \mathrm{CDCl}_{3}, 25{ }^{\circ} \mathrm{C}\right): \delta=166.5,155.7$, $152.9,136.9,136.0,133.7,129.7,127.5,125.7,122.16,113.5,96.7,26.3,12.2$. EIMS: $m / z(\%)=$ $386[\mathrm{M}]^{+}$(100), 351 (54.31), 296 (13.34), 270 (5.79), 253 (6.49), 213 (16.38), 67 (41.21). HRMass(EI): $m / z$ Calcd for $\mathrm{C}_{16} \mathrm{H}_{11} \mathrm{~F}_{3} \mathrm{Cl}_{2} \mathrm{~N}_{4}$ : 386.0313; found: 386.0286 .

1-(2,6-Dichloro-4-(trifluoromethyl)phenyl)-5-((E)-((E)-hex-2-enylidene)amino)-1H-pyrazole-3carbonitrile (11). Mp 102.4 - $103.0{ }^{\circ} \mathrm{C}$. IR (KBr): 3125, 2248, 1640, 1585, 1313, 1137, 822, 670 
$\mathrm{cm}^{-1} .{ }^{1} \mathrm{H}$ NMR $\left(400 \mathrm{MHz}, \mathrm{CDCl}_{3}, 25{ }^{\circ} \mathrm{C}\right): \delta=0.94\left(\mathrm{t}, J=7.2 \mathrm{~Hz}, 3 \mathrm{H}, \mathrm{CH}_{3}\right), 1.54-1.49(\mathrm{~m}, 2 \mathrm{H}$, $\left.\mathrm{CH}_{2}\right)$, 2.30-2.24 (m, $\left.2 \mathrm{H}, \mathrm{CH}_{2}\right), 6.31-6.25$ (m, $\left.1 \mathrm{H}, \mathrm{CH}=\mathrm{CH}\right), 6.66-6.59(\mathrm{~m}, 1 \mathrm{H}, \mathrm{CH}), 6.60(\mathrm{~s}, 1 \mathrm{H}$, Pyrazole-H), 7.73 (s, $2 \mathrm{H}, \mathrm{ArH}), 8.26$ (d, $J=9.2 \mathrm{~Hz}, 1 \mathrm{H}, \mathrm{N}=\mathrm{CH}) .{ }^{13} \mathrm{C} \mathrm{NMR}\left(100 \mathrm{MHz}, \mathrm{CDCl}_{3}, 25\right.$ $\left.{ }^{\circ} \mathrm{C}\right): \delta=166.4,154.3,152.9,136.9,136.0,133.5,130.7,127.5,125.6,122.1,113.5,96.7,35.2,21.4$, 13.6. EIMS: $m / z(\%)=400[\mathrm{M}]^{+}(100), 385$ (54.48), 371 (50.78), 357 (36.50), 323 (37.49), 296 (16.61), 271 (28.58), 213 (29.29), 81 (88.13). HRMass(EI): $m / z$ Calcd for $\mathrm{C}_{17} \mathrm{H}_{13} \mathrm{~F}_{3} \mathrm{Cl}_{2} \mathrm{~N}_{4}: 400.0469$; found: 400.0470 .

1-(2,6-Dichloro-4-(trifluoromethyl)phenyl)-5-((E)-((E)-hept-2-enylidene)amino)-1H-pyrazole3-carbonitrile (12). Mp 93.1 - $94.0^{\circ} \mathrm{C}$. IR (KBr): 3126, 2249, 1642, 1588, 1314, 1138, 822, 677 $\mathrm{cm}^{-1} .{ }^{1} \mathrm{H}$ NMR $\left(400 \mathrm{MHz}, \mathrm{CDCl}_{3}, 25^{\circ} \mathrm{C}\right): \delta=1.00-0.95\left(\mathrm{~m}, 3 \mathrm{H}, \mathrm{CH}_{3}\right), 1.54-1.32\left(\mathrm{~m}, 4 \mathrm{H}, \mathrm{CH}_{2} \mathrm{X} 2\right)$, 2.32-2.26 (m, $\left.2 \mathrm{H}, \mathrm{CH}_{2}\right), 6.31-6.25$ (m, $\left.1 \mathrm{H}, \mathrm{CH}=\mathrm{CH}\right), 6.60$ (s, $1 \mathrm{H}$, Pyrazole-H), 6.66-6.59 (m, $1 \mathrm{H}$, $\mathrm{CH}), 7.73$ (s, $2 \mathrm{H}, \mathrm{ArH}), 8.26(\mathrm{~d}, J=9.2 \mathrm{~Hz}, 1 \mathrm{H}, \mathrm{N}=\mathrm{CH}) .{ }^{13} \mathrm{C} \mathrm{NMR}\left(100 \mathrm{MHz}, \mathrm{CDCl}_{3}, 25{ }^{\circ} \mathrm{C}\right): \delta=$ $166.5,154.4,152.9,136.9,136.0,133.9,130.6,127.5,125.6,122.1,113.5,96.7,32.9,30.2,22.2$, 13.8. EIMS: $m / z(\%)=414[\mathrm{M}]^{+}$(27.12), 385 (100), 357 (91.55), 323 (31.03), 296 (16.58), 271 (21.16), 213 (22.67), 95 (24.68). HRMass(EI): $m / z$ Calcd for $\mathrm{C}_{18} \mathrm{H}_{15} \mathrm{~F}_{3} \mathrm{Cl}_{2} \mathrm{~N}_{4}$ : 414.0626; found: 414.0627.

1-(2,6-Dichloro-4-(trifluoromethyl)phenyl)-5-((E)-((E)-3-phenylallylidene)amino)-1H-

pyrazole-3-carbonitrile (13). $\mathrm{Mp} 156.8$ - $157.2^{\circ} \mathrm{C}$. IR (KBr): 3100, 2239, 1583, 1310, 1192. 830 $\mathrm{cm}^{-1} .{ }^{1} \mathrm{H}$ NMR $\left(400 \mathrm{MHz}, \mathrm{CDCl}_{3}, 25^{\circ} \mathrm{C}\right): \delta=6.71(\mathrm{~d}, J=4.4 \mathrm{~Hz}, 1 \mathrm{H}, \mathrm{CH}=\mathrm{CH}), 6.91-6.98(\mathrm{~m}, 1 \mathrm{H}$, $\mathrm{CH}=\mathrm{CH}), 7.26(\mathrm{~d}, J=3.6 \mathrm{~Hz}, 1 \mathrm{H}, \mathrm{CH}), 7.39-7.40(\mathrm{~m}, 3 \mathrm{H}, \mathrm{ArH}), 7.50-7.52(\mathrm{~m}, 2 \mathrm{H}, \mathrm{ArH}), 7.75(\mathrm{~s}$, $2 \mathrm{H}, \mathrm{ArH}), 8.44$ (d, $J=9.2 \mathrm{~Hz}, 1 \mathrm{H}, \mathrm{N}=\mathrm{CH}) .{ }^{13} \mathrm{C} \mathrm{NMR}\left(100 \mathrm{MHz}, \mathrm{CDCl}_{3}, 25{ }^{\circ} \mathrm{C}\right): \delta=165.7,152.9$, 148.6, 137.0, 136.0, 134.8, 133.8, 130.7, 129.0, 128.0, 127.6, 125.7, 125.6, 122.2, 113.4, 96.8. EIMS: $m / z(\%)=433[\mathrm{M}]^{+}$(96.38), 399 (9.69), 217 (2.51), 213 (9.57), 178 (3.10), 168 (4.59), 143 (2.97), 129 (3.86), 115 (100.00), 103 (3.52), 89 (5.71), 65 (2.34), 51 (2.66). HRMass(EI): m/z Calcd for $\mathrm{C}_{20} \mathrm{H}_{11} \mathrm{~F}_{3} \mathrm{Cl}_{2} \mathrm{~N}_{4}$ : 433.0235 ; found: 433.0206 .

1-(2,6-Dichloro-4-(trifluoromethyl)phenyl)-4,5,6,7-tetrahydro-1H-pyrazolo[3,4-b]pyridine-3-

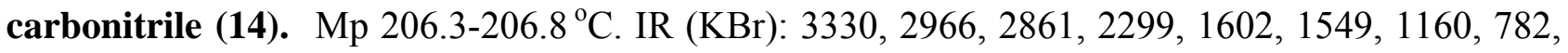
$678 \mathrm{~cm}^{-1} .{ }^{1} \mathrm{H}$ NMR $\left(400 \mathrm{MHz}, \mathrm{CDCl}_{3}, 25{ }^{\circ} \mathrm{C}\right): \delta=1.93-1.95\left(\mathrm{~m}, 2 \mathrm{H}, \mathrm{CH}_{2}\right), 2.72(\mathrm{t}, J=6.3 \mathrm{~Hz}, 2 \mathrm{H}$, $\left.\mathrm{CH}_{2}\right), 3.35\left(\mathrm{t}, J=5.4 \mathrm{~Hz}, 2 \mathrm{H}, \mathrm{CH}_{2}\right), 3.50(\mathrm{~s}, 1 \mathrm{H}, \mathrm{NH}), 7.72$ (s, $\left.2 \mathrm{H}, \mathrm{ArH}\right) .{ }^{13} \mathrm{C} \mathrm{NMR}(100 \mathrm{MHz}$, $\left.\mathrm{CDCl}_{3}, 25{ }^{\circ} \mathrm{C}\right): \delta=145.8,136.3,135.8,133.9,126.1,125.9,122.0,113.4,104.1,42.6,21.8,18.4$. EIMS: $m / z(\%)=360[\mathrm{M}]^{+}$(100.00), 325 (12.06), 297 (29.66), 273 (5.35), 215 (8.60), 213 (14.13), 178 (6.23), 133 (19.18). HRMass(EI): $m / z$ Calcd for $\mathrm{C}_{14} \mathrm{H}_{9} \mathrm{~F}_{3} \mathrm{Cl}_{2} \mathrm{~N}_{4}$ : 360.0156; found: 360.0176.

1-(2,6-Dichloro-4-(trifluoromethyl)phenyl)-6-methyl-4,5,6,7-tetrahydro-1H-pyrazolo[3,4b]pyridine-3-carbonitrile (15). $\mathrm{Mp} 232.1-233.2^{\circ} \mathrm{C}$. IR (KBr): 3392, 2964, 2938, 2842, 2249, 1592, $1541,1314,1142,885,709 \mathrm{~cm}^{-1} .{ }^{1} \mathrm{H} \mathrm{NMR}\left(400 \mathrm{MHz}, \mathrm{CDCl}_{3}, 25^{\circ} \mathrm{C}\right): \delta=1.24(\mathrm{~d}, J=4.8 \mathrm{~Hz}, 3 \mathrm{H}$, $\left.\mathrm{CH}_{3}\right), 1.51-1.60\left(\mathrm{~m}, 1 \mathrm{H}, \mathrm{CH}_{2}\right), 1.98-2.01\left(\mathrm{~m}, 1 \mathrm{H}, \mathrm{CH}_{2}\right), 2.65-2.78\left(\mathrm{~m}, 2 \mathrm{H}, \mathrm{CH}_{2}\right), 3.31(\mathrm{~s}, 1 \mathrm{H}, \mathrm{NH})$, 3.45 (m, $1 \mathrm{H}, \mathrm{CH}), 7.75$ (s, $2 \mathrm{H}, \mathrm{ArH}) .{ }^{13} \mathrm{C} \mathrm{NMR}\left(100 \mathrm{MHz}, \mathrm{CDCl}_{3}, 25{ }^{\circ} \mathrm{C}\right): \delta=146.0,136.3,135.8$, 134.2, 126.1, 124.6, 113.5, 109.1, 103.8, 48.8, 29.4, 21.1, 17.6. EIMS: $m / z(\%)=374[\mathrm{M}]^{+}(52.25)$, 361 (58.88), 359 (100.00), 339 (3.27), 333 (5.09), 323 (3.84), 297 (8.10), 215 (5.16), 213 (8.53), 178 (3.40), 143 (2.48), 41 (2.42). HRMass(EI): $m / z$ Calcd for $\mathrm{C}_{15} \mathrm{H}_{11} \mathrm{~F}_{3} \mathrm{Cl}_{2} \mathrm{~N}_{4}$ : 374.0313; found: 374.0324 . 
1-(2,6-Dichloro-4-(trifluoromethyl)phenyl)-6-ethyl-4,5,6,7-tetrahydro-1H-pyrazolo[3,4b]pyridine-3-carbonitrile (16). Mp 181.1-181.7 ${ }^{\circ} \mathrm{C}$. IR (KBr): 3393, 2965, 2933, 2848, 2249, 1593, 1541, 1319, 1142, 884, $710 \mathrm{~cm}^{-1} .{ }^{1} \mathrm{H}$ NMR (400 MHz, $\left.\mathrm{CDCl}_{3}, 25{ }^{\circ} \mathrm{C}\right): \delta=0.96(\mathrm{t}, J=7.6 \mathrm{~Hz}, 3 \mathrm{H}$, $\left.\mathrm{CH}_{3}\right), 1.22-1.30(\mathrm{~m}, 1 \mathrm{H}, \mathrm{CH}), 1.52-1.57\left(\mathrm{~m}, 2 \mathrm{H}, \mathrm{CH}_{2}\right), 1.6-1.65\left(\mathrm{~m}, 1 \mathrm{H}, \mathrm{CH}_{2}\right), 2.00-2.15(\mathrm{~m}, 1 \mathrm{H}$, $\left.\mathrm{CH}_{2}\right), 2.68-2.74(\mathrm{~m}, 1 \mathrm{H}, \mathrm{CH}), 3.24(\mathrm{~d}, J=7.2 \mathrm{~Hz}, 1 \mathrm{H}, \mathrm{CH}), 3.41(\mathrm{~s}, 1 \mathrm{H}, \mathrm{NH}), 7.76$ (s, $\left.2 \mathrm{H}, \mathrm{ArH}\right)$. ${ }^{13} \mathrm{C}$ NMR $\left(100 \mathrm{MHz}, \mathrm{CDCl}_{3}, 25{ }^{\circ} \mathrm{C}\right): \delta=145.9,136.4,136.2,135.8,134.2,133.9,126.1,125.7$, 113.5, 103.8, 54.5, 27.8, 26.8, 17.4, 10.1. EIMS: $m / z(\%)=388[\mathrm{M}]^{+}$(28.99), 359 (100), 324 (4.11), 213 (4.86), 69 (5.74), 41 (4.74). HRMass(EI): $m / z$ Calcd for $\mathrm{C}_{16} \mathrm{H}_{13} \mathrm{~F}_{3} \mathrm{Cl}_{2} \mathrm{~N}_{4}$ : 388.0469; found: 388.0469 .

\section{1-(2,6-Dichloro-4-(trifluoromethyl)phenyl)-6-propyl-4,5,6,7-tetrahydro-1H-pyrazolo[3,4-}

b]pyridine-3-carbonitrile (17). Mp 173.9 - $174.6{ }^{\circ} \mathrm{C}$. IR (KBr): 3395, 2968, 2935, 2843, 2249, $1591,1541,1311,1146,883,710 \mathrm{~cm}^{-1} .{ }^{1} \mathrm{H} \mathrm{NMR}\left(400 \mathrm{MHz}, \mathrm{CDCl}_{3}, 25{ }^{\circ} \mathrm{C}\right): \delta=0.92(\mathrm{t}, J=7.2 \mathrm{~Hz}$, $\left.3 \mathrm{H}, \mathrm{CH}_{3}\right), 1.51-1.37$ (m, $4 \mathrm{H}, \mathrm{CH}_{2} \mathrm{X}$ 2), 1.68-1.63 (m, $\left.1 \mathrm{H}, \mathrm{CH}\right), 2.02-1.99$ (m, $\left.1 \mathrm{H}, \mathrm{CH}_{2}\right), 2.76-$ $2.65\left(\mathrm{~m}, 2 \mathrm{H}, \mathrm{CH}_{2}\right), 3.60-3.20(\mathrm{~m}, 2 \mathrm{H}, \mathrm{NH}$ and $\mathrm{CH}), 7.56(\mathrm{~s}, 2 \mathrm{H}, \mathrm{ArH}) .{ }^{13} \mathrm{C} \mathrm{NMR}(100 \mathrm{MHz}$, $\left.\mathrm{CDCl}_{3}, 25{ }^{\circ} \mathrm{C}\right): \delta=145.9,136.4,136.2,133.9,126.1,123.4,120.7,113.6,103.8,52.8,37.1,27.3$, 19.0, 17.4, 14.0. EIMS: $m / z(\%)=402[\mathrm{M}]^{+}$(22.62), 359 (100), 332 (3.33), 213 (3.57). HRMass(EI): $m / z$ Calcd for $\mathrm{C}_{17} \mathrm{H}_{15} \mathrm{~F}_{3} \mathrm{Cl}_{2} \mathrm{~N}_{4}$ : 402.0626; found: 402.0626 .

6-Butyl-1-(2,6-dichloro-4-(trifluoromethyl)phenyl)-4,5,6,7-tetrahydro-1H-pyrazolo[3,4b]pyridine-3-carbonitrile (18). $\mathrm{Mp} 116.5$ - $117.0{ }^{\circ} \mathrm{C}$. IR (KBr): 3393, 2966, 2934, 2842, 2245 , 1590, 1541, 1313, 1140, 883, $\left.709 \mathrm{~cm}^{-1} .{ }^{1} \mathrm{H} \mathrm{NMR} \mathrm{(400} \mathrm{MHz,} \mathrm{CDCl}_{3}, 25{ }^{\circ} \mathrm{C}\right): \delta=0.90\left(\mathrm{~s}, 3 \mathrm{H}, \mathrm{CH}_{3}\right)$, 1.28-1.32 (m, $4 \mathrm{H}, \mathrm{CH}_{2} \mathrm{X}$ 2), 1.47-1.51 (m, $\left.2 \mathrm{H}, \mathrm{CH}_{2}\right), 1.68-1.63$ (m, $\left.1 \mathrm{H}, \mathrm{CH}_{2}\right), 2.02-2.00(\mathrm{~m}, 1 \mathrm{H}$, $\left.\mathrm{CH}_{2}\right), 2.76-2.67\left(\mathrm{~m}, 2 \mathrm{H}, \mathrm{CH}_{2}\right), 3.20-3.36(\mathrm{~m}, 2 \mathrm{H}, \mathrm{NH}$ and $\mathrm{CH}), 7.76(\mathrm{~s}, 2 \mathrm{H}, \mathrm{ArH}) .{ }^{13} \mathrm{C}$ NMR $(100$ $\left.\mathrm{MHz}, \mathrm{CDCl}_{3}, 25^{\circ} \mathrm{C}\right): \delta=145.9,136.4,136.2,135.1,126.1,125.7,122.1,113.5,103.8,55.1,34.6$, 27.9, 27.3, 22.6, 17.4, 13.9. EIMS: $m / z(\%)=416[\mathrm{M}]^{+}$(25.30), 359 (100), 333 (4.27), 324 (3.09). HRMass(EI): $m / z$ Calcd for $\mathrm{C}_{18} \mathrm{H}_{17} \mathrm{~F}_{3} \mathrm{Cl}_{2} \mathrm{~N}_{4}$ : 416.0782; found: 416.0782.

1-(2,6-Dichloro-4-(trifluoromethyl)phenyl)-1H-pyrazolo[3,4-b]pyridine-3-carbonitrile (19). Mp 148.3-149.8 ${ }^{\circ} \mathrm{C}$. IR (KBr): 3090, 2918, 2842, 2239, $1592 \mathrm{~cm}^{-1} .{ }^{1} \mathrm{H}$ NMR (400 MHz, $\left.\mathrm{CDCl}_{3}, 25{ }^{\circ} \mathrm{C}\right)$ : $\delta=7.50(\mathrm{t}, J=4.5 \mathrm{~Hz}, 1 \mathrm{H}$, Pyridine-H), 7.84 (s, $2 \mathrm{H}, \mathrm{ArH}), 8.35$ (d, $J=8.2 \mathrm{~Hz}, 1 \mathrm{H}$, Pyridine-H), $8.71\left(\mathrm{~d}, J=4.5 \mathrm{~Hz}, 1 \mathrm{H}\right.$, Pyridine-H). ${ }^{13} \mathrm{C} \mathrm{NMR}\left(100 \mathrm{MHz}, \mathrm{CDCl}_{3}, 25{ }^{\circ} \mathrm{C}\right): \delta=151.7,150.7,136.5$, $135.7,134.4,129.5,126.1,122.1,121.1,120.5,116.6,112.0$. EIMS: $m / z(\%)=356[\mathrm{M}]^{+}(11.73)$, 321 (100.00), 269 (19.83), 241 (9.77), 217 (5.76), 143 (7.54). HRMass(EI): $m / z$ Calcd for $\mathrm{C}_{14} \mathrm{H}_{5} \mathrm{~F}_{3} \mathrm{Cl}_{2} \mathrm{~N}_{4}$ : 355.9843; found: 355.9850 .

1-(2,6-Dichloro-4-(trifluoromethyl)phenyl)-6-methyl-1H-pyrazolo[3,4-b]pyridine-3-

carbonitrile (20). $\mathrm{Mp} 141.8-142.5^{\circ} \mathrm{C}$. IR (KBr): 3040, 2240, 1594, 1577, 1315, 1124, $871 \mathrm{~cm}^{-1} .{ }^{1} \mathrm{H}$ NMR (400 MHz, $\left.\mathrm{CDCl}_{3}, 25{ }^{\circ} \mathrm{C}\right): \delta=2.68\left(\mathrm{~s}, 3 \mathrm{H}, \mathrm{CH}_{3}\right), 7.32(\mathrm{~d}, J=8.3 \mathrm{~Hz}, 1 \mathrm{H}$, Pyridine-H), 7.82 (s, $2 \mathrm{H}, \mathrm{ArH}), 8.17$ (d, $J=8.3 \mathrm{~Hz}, 1 \mathrm{H}$, Pyridine-H). ${ }^{13} \mathrm{C} \mathrm{NMR}\left(100 \mathrm{MHz}, \mathrm{CDCl}_{3}, 25{ }^{\circ} \mathrm{C}\right): \delta=162.4$, $150.9,136.5,136.0,134.3,128.9,126.0,121.2,120.8,114.5,112.3,25.0$. EIMS: $\mathrm{m} / z(\%)=370$ $\left[\mathrm{M}^{+}\right.$(7.33), 335 (100.00), 301 (1.55), 283 (3.14), 247 (4.36), 213 (3.83), 179 (11.27), 143 (3.47), 51 (2.56). HRMass(EI): $m / z$ Calcd for $\mathrm{C}_{15} \mathrm{H}_{7} \mathrm{~F}_{3} \mathrm{Cl}_{2} \mathrm{~N}_{4}$ : 370.0000 ; found: 370.0002 .

1-(2,6-Dichloro-4-(trifluoromethyl)phenyl)-6-ethyl-1H-pyrazolo[3,4-b]pyridine-3-carbonitrile (21). Mp 125.6 - $126.3{ }^{\circ} \mathrm{C}$. IR (KBr): $3041,2237,1597,1579,1313,1125,871 \mathrm{~cm}^{-1}$. ${ }^{1} \mathrm{H}$ NMR (400 
$\left.\mathrm{MHz}, \mathrm{CDCl}_{3}, 25{ }^{\circ} \mathrm{C}\right): \delta=1.31\left(\mathrm{t}, J=7.6 \mathrm{~Hz}, 3 \mathrm{H}, \mathrm{CH}_{3}\right), 2.94\left(\mathrm{q}, J=7.6 \mathrm{~Hz}, 2 \mathrm{H}, \mathrm{CH}_{2}\right), 7.34(\mathrm{~d}, J=$ $8.4 \mathrm{~Hz}, 1 \mathrm{H}$, Pyridine-H), 7.83 (s, $2 \mathrm{H}, \mathrm{ArH}), 8.19$ (d, J=8.0 Hz, $1 \mathrm{H}$, Pyridine-H). ${ }^{13} \mathrm{C}$ NMR (100 $\left.\mathrm{MHz}, \mathrm{CDCl}_{3}, 25^{\circ} \mathrm{C}\right): \delta=167.4,150.9,136.5,136.1,134.2,129.0,126.1,126.0,120.9,120.1,114.7$, 112.3, 31.8, 13.8. EIMS: $m / z(\%)=384[\mathrm{M}]^{+}$(100.00), 349 (47.06), 334 (7.69), 247 (4.63), 213 (4.23). HRMass(EI): $m / z$ Calcd for $\mathrm{C}_{16} \mathrm{H}_{9} \mathrm{~F}_{3} \mathrm{Cl}_{2} \mathrm{~N}_{4}$ : 384.0156 ; found: 384.0156 .

\section{1-(2,6-Dichloro-4-(trifluoromethyl)phenyl)-6-propyl-1H-pyrazolo[3,4-b]pyridine-3-}

carbonitrile (22). Mp 105.0 - $105.5^{\circ} \mathrm{C}$. IR (KBr): 3047, 2236, 1593, 1578, 1313, $1125,871 \mathrm{~cm}^{-1}$. ${ }^{1} \mathrm{H}$ NMR $\left(400 \mathrm{MHz}, \mathrm{CDCl}_{3}, 25{ }^{\circ} \mathrm{C}\right): \delta=0.95\left(\mathrm{t}, J=7.2 \mathrm{~Hz}, 3 \mathrm{H}, \mathrm{CH}_{3}\right), 1.74-1.79\left(\mathrm{~m}, 2 \mathrm{H}, \mathrm{CH}_{2}\right)$, $2.88\left(\mathrm{t}, J=7.6 \mathrm{~Hz}, 2 \mathrm{H}, \mathrm{CH}_{2}\right), 7.32$ (d, $J=8.4 \mathrm{~Hz}, 1 \mathrm{H}$, Pyridine-H), 7.83 (s, $\left.2 \mathrm{H}, \mathrm{ArH}\right), 8.18$ (d, $J=$ $8.0 \mathrm{~Hz}, 1 \mathrm{H}$, Pyridine-H). ${ }^{13} \mathrm{C} \mathrm{NMR}\left(100 \mathrm{MHz}, \mathrm{CDCl}_{3}, 25{ }^{\circ} \mathrm{C}\right): \delta=166.3,150.9,136.5,136.0$, 134.2, 128.9, 126.3, 126.0, 123.5, 120.7, 114.7, 112.3, 40.5, 23.0, 13.7. EIMS: $m / z(\%)=398$ [M] $^{+}$ (3.55), 383 (17.66), 370 (100), 335 (7.99), 247 (4.20), 213 (2.77). HRMass(EI): $m / z$ Calcd for $\mathrm{C}_{17} \mathrm{H}_{11} \mathrm{~F}_{3} \mathrm{Cl}_{2} \mathrm{~N}_{4}$ : 398.0313; found: 398.0313 .

6-Butyl-1-(2,6-dichloro-4-(trifluoromethyl)phenyl)-1H-pyrazolo[3,4-b]pyridine-3-carbonitrile (23). Mp 58.5 - 59.4 ${ }^{\circ} \mathrm{C}$. IR (KBr): 3047, 2235, 1598, 1572, 1311, 1126, $871 \mathrm{~cm}^{-1}$. ${ }^{1} \mathrm{H}$ NMR (400 $\left.\mathrm{MHz}, \mathrm{CDCl}_{3}, 25^{\circ} \mathrm{C}\right): \delta=0.92\left(\mathrm{t}, J=7.2 \mathrm{~Hz}, 3 \mathrm{H}, \mathrm{CH}_{2}\right), 1.32-1.39\left(\mathrm{~m}, 2 \mathrm{H}, \mathrm{CH}_{2}\right), 1.67-1.73(\mathrm{~m}, 2 \mathrm{H}$, $\mathrm{CH}_{2}$ ), 2.90 (t, $\left.J=8.0 \mathrm{~Hz}, 2 \mathrm{H}, \mathrm{CH}_{2}\right), 7.33$ (d, $J=8.4 \mathrm{~Hz}, 1 \mathrm{H}$, Pyridine-H), 7.82 (s, $\left.2 \mathrm{H}, \mathrm{ArH}\right), 8.18$ $\left(\mathrm{d}, J=8.4 \mathrm{~Hz}, 1 \mathrm{H}\right.$, Pyridine-H). ${ }^{13} \mathrm{C} \mathrm{NMR}\left(100 \mathrm{MHz}, \mathrm{CDCl}_{3}, 25{ }^{\circ} \mathrm{C}\right): \delta=166.6,150.9,136.4$, 136.1, 134.2, 128.9, 126.0, 123.5, 120.8, 120.6, 114.6, 112.3, 38.3, 31.8, 22.3, 13.8. EIMS: $\mathrm{m} / z(\%)$ $=412[\mathrm{M}]^{+}$(3.55), 383 (10.32), 370 (100), 335 (6.60), 247 (2.57). HRMass(EI): $m / z$ Calcd for $\mathrm{C}_{18} \mathrm{H}_{13} \mathrm{~F}_{3} \mathrm{Cl}_{2} \mathrm{~N}_{4}$ : 412.0469; found: 412.0467.

1-(2,6-Dichloro-4-(trifluoromethyl)phenyl)-6-phenyl-1H-pyrazolo[3,4-b]pyridine-3-

carbonitrile (24). $\mathrm{Mp} 172.3-174.1^{\circ} \mathrm{C}$. IR (KBr): 3071, 2918, 2239, 1597, 1319, 1128, $830 \mathrm{~cm}^{-1} .{ }^{1} \mathrm{H}$ NMR (400 MHz, $\left.\mathrm{CDCl}_{3}, 25^{\circ} \mathrm{C}\right): \delta=7.44-7.48$ (m, $\left.3 \mathrm{H}, \mathrm{ArH}\right), 7.85$ (s, $\left.2 \mathrm{H}, \mathrm{ArH}\right), 7.93(\mathrm{~d}, J=8.5$ $\mathrm{Hz}, 1 \mathrm{H}$, Pyridine-H), 8.01-8.05 (m, $2 \mathrm{H}, \mathrm{ArH}), 8.35$ (d, $J=8.5 \mathrm{~Hz}, 1 \mathrm{H}$, Pyridine-H). ${ }^{13} \mathrm{C}$ NMR $\left(100 \mathrm{MHz}, \mathrm{CDCl}_{3}, 25^{\circ} \mathrm{C}\right): \delta=159.9,151.1,137.7,136.4,136.0,134.1,130.5,129.7,129.0,127.8$, 126.1, 126.0, 121.0, 118.2, 115.2, 112.2. EIMS: $m / z(\%)=432[\mathrm{M}]^{+}(18.32), 397(100.00), 344$ (9.58), 310 (10.77), 213 (3.65), 178 (4.34), 166 (2.41), 143 (3.91), 140 (12.60), 115 (13.98), 77 (5.65), 64 (4.09), 51 (4.02). HRMass(EI): $m / z$ Calcd for $\mathrm{C}_{20} \mathrm{H}_{9} \mathrm{~F}_{3} \mathrm{Cl}_{2} \mathrm{~N}_{4}$ : 432.0156; found: 432.0156. (Z)-1-(2,6-Dichloro-4-(trifluoromethyl)phenyl)-6-phenyl-4-((E)-3-phenylallylidene)-4,7dihydro-1H-pyrazolo[3,4-b]pyridine-3-carbonitrile (25). Mp 225.0 - $225.3{ }^{\circ} \mathrm{C}$. IR (KBr): 3071 , 2225, 1590, 1550, 1320, 1128, $830 \mathrm{~cm}^{-1} .{ }^{1} \mathrm{H} \mathrm{NMR}$ (400 MHz, $\left.\mathrm{CDCl}_{3}, 25{ }^{\circ} \mathrm{C}\right): \delta=5.44(\mathrm{~s}, 1 \mathrm{H}, \mathrm{CH})$, $6.88(\mathrm{~d}, J=15.2 \mathrm{~Hz}, 1 \mathrm{H}, \mathrm{CH}=\mathrm{CH}), 6.94(\mathrm{~d}, J=2.4 \mathrm{~Hz}, 1 \mathrm{H}, \mathrm{CH}=\mathrm{CH}), 7.02-7.07(\mathrm{~m}, 1 \mathrm{H}, \mathrm{CH}=\mathrm{CH})$, 7.28-7.40 (m, $10 \mathrm{H}, \mathrm{ArH}), 7.77$ (s, $2 \mathrm{H}, \mathrm{ArH}), 7.85$ (s, $1 \mathrm{H}, \mathrm{NH}) .{ }^{13} \mathrm{C} \mathrm{NMR}\left(100 \mathrm{MHz}, \mathrm{CDCl}_{3}, 25\right.$ $\left.{ }^{\circ} \mathrm{C}\right): \delta=164.2,146.8,146.0,143.8,143.5,136.1,136.0,135.7,132.5,130.0,129.4,129.0,127.9$, $127.8,127.6,127.3,125.9,125.8,125.7,125.0,124.9,113.0,112.5$. EIMS: $m / z(\%)=548[\mathrm{M}]^{+}$, 457 (35.80), 421 (8.98), 409 (6.80), 92 (100), 77 (6.19). HRMass(EI): $m / z$ Calcd for $\mathrm{C}_{29} \mathrm{H}_{17} \mathrm{~F}_{3} \mathrm{Cl}_{2} \mathrm{~N}_{4}$ : 548.0782; found: 548.0828 .

1-(2,6-Dichloro-4-(trifluoromethyl)phenyl)-6-ethyl-5-methyl-1H-pyrazolo[3,4-b]pyridine-3carbonitrile (27). $\mathrm{Mp} 108.5-109.3{ }^{\circ} \mathrm{C}$. IR (KBr): 3045, 2233, 1591, 1577, 1311, 1127, $871 \mathrm{~cm}^{-1} .{ }^{1} \mathrm{H}$ NMR (400 MHz, $\left.\mathrm{CDCl}_{3}, 25{ }^{\circ} \mathrm{C}\right): \delta=1.25\left(\mathrm{t}, J=7.6 \mathrm{~Hz}, 3 \mathrm{H}, \mathrm{CH}_{3}\right), 2.52\left(\mathrm{~s}, 3 \mathrm{H}, \mathrm{CH}_{3}\right), 2.83-2.90$ 
(m, $\left.2 \mathrm{H}, \mathrm{CH}_{2}\right), 7.82(\mathrm{~s}, 2 \mathrm{H}, \mathrm{ArH}), 7.98$ (s, $1 \mathrm{H}$, Pyridine-H). ${ }^{13} \mathrm{C}$ NMR $\left(100 \mathrm{MHz}, \mathrm{CDCl}_{3}, 25^{\circ} \mathrm{C}\right): \delta$ $=166.1,149.8,136.4,136.2,134.1,129.1,128.5,126.0,125.9,123.6,120.8,120.0,115.0,112.5$, 29.2, 19.2, 12.2. EIMS: $m / z(\%)=398[\mathrm{M}]^{+}(100.00), 363$ (30.86), 335 (8.70); HRMass(EI): $m / z$ Calcd for $\mathrm{C}_{17} \mathrm{H}_{11} \mathrm{~F}_{3} \mathrm{Cl}_{2} \mathrm{~N}_{4}$ : 398.0313 ; found: 398.0313 .

1-(2,6-Dichloro-4-(trifluoromethyl)phenyl)-5-((E)-((E)-2-methylpent-2-enylidene)amino)-1Hpyrazole-3-carbonitrile (28). Mp $110.9-111.5^{\circ} \mathrm{C}$. IR (KBr): 3129, 2248, 1640, 1588, 1314, 1133 , $822,675 \mathrm{~cm}^{-1} .{ }^{1} \mathrm{H}$ NMR $\left(400 \mathrm{MHz}, \mathrm{CDCl}_{3}, 25^{\circ} \mathrm{C}\right): \delta=1.09\left(\mathrm{t}, J=7.2 \mathrm{~Hz}, 3 \mathrm{H}, \mathrm{CH}_{3}\right), 1.67(\mathrm{~s}, 3 \mathrm{H}$, $\left.\mathrm{CH}_{3}\right), 2.31-2.35\left(\mathrm{~m}, 2 \mathrm{H}, \mathrm{CH}_{2}\right), 6.28(\mathrm{t}, J=6.8 \mathrm{~Hz}, 1 \mathrm{H}, \mathrm{CH}), 6.60(\mathrm{~s}, 1 \mathrm{H}$, Pyrazole-H), $7.74(\mathrm{~s}, 2 \mathrm{H}$, $\mathrm{ArH}), 8.20(\mathrm{~s}, 1 \mathrm{H}, \mathrm{N}=\mathrm{CH}) .{ }^{13} \mathrm{C} \mathrm{NMR}\left(100 \mathrm{MHz}, \mathrm{CDCl}_{3}, 25{ }^{\circ} \mathrm{C}\right): \delta=168.8,152.9,152.5,137.0$, 136.1, 135.9, 133.6, 127.4, 125.5, 122.2, 113.6, 96.5, 22.6, 13.1, 10.6. EIMS: $m / z(\%)=400[\mathrm{M}]^{+}$ (100), 385 (58.18), 365 (16.81), 349 (6.72), 296 (9.95), 213 (12.41), 81 (34.27), 41 (8.44). HRMass(EI): $m / z$ Calcd for $\mathrm{C}_{17} \mathrm{H}_{13} \mathrm{~F}_{3} \mathrm{Cl}_{2} \mathrm{~N}_{4}$ : 400.0469; found: 400.0470 .

\section{Acknowledgements}

This work was financial supported by National High Technology Research and Development Program of China (863 Program, 2006AA10A201). The authors also appreciate the partly support from Shanghai Foundation of Science and Technology (073919107, 064319022), Shanghai Leading Academic Discipline Project, Project Number: B507.

\section{References}

1. (a) Hardy, C. R. Adv. Heterocycl. Chem. 1984, 36, 343. (b) Yu, G.; Mason, H.; Wu, X.; Wang, J.; Chong, S.; Dorough, G.; Henwood, A.; Pongrac, R.; Seliger, L.; He, B.; Normandin, D.; Adam, L.; Krupinski, J.; Macor, J. J. Med. Chem. 2001, 44, 1025. (c) El-Ahl, A. A. S.; Metwally, M. A.; Amer, F. A. Boll. Chim. Farm. 1995, 7, 369.

2. (a) Bettinetti, L.; Schlotter, K.; Hübner, H.; Gmeiner, P. J. Med. Chem. 2002, 45, 4594. (b) Löber, S.; Hübner, H.; Utz, W.; Gmeiner, P. J. Med. Chem. 2001, 44, 2691. (c) Kuroda, S.; Akahane, A.; Itani, H.; Nishimura, S.; Durkin, K.; Tenda, Y.; Sakane, K. Bioorg. Med. Chem. 2000, 8, 55. (d) Johns, B. A.; Gudmundsson,; Turner, K. S. E. M.; Allen, S. H.; Samano, V. A.; Ray, J. A.; Freeman, G. A.; Boyd, F. L.; Sexton, C. J.; Selleseth, D. W.; Creech, K. L.; Moniri, K. R. Bioorg. Med. Chem. 2005, 13, 2397. (e) Gudmundsson, K. S.; Johns, B. A.; Wang, Z.; Turner, E. M.; Allen, S. H.; Freeman, G. A.; Jr, F. L. B.; Sexton, C. J.; Selleseth, D. W.; Monirib, K. R.; Creech, K. L. Bioorg. Med. Chem. 2005, 13, 5346.

3. (a) Harju, K.; Kylänlahti, I.; Paananen, T.; Polamo, M.; Nielsen, J.; Yli-Kauhaluoma, J. J. Comb. Chem. 2006, 8, 344. (b) Mshvidobadze, E. V.; Vasilevskya, S. F.; Elguero, J. Tetrahedron 2004, 60, 11875. (c) Díaz-Ortiz, A.; de la. Hoz, A.; Langab, F. Green Chemistry 2000, 2, 165.

4. (a) Abonia, R.; Rengifo, E.; Quiroga, J.; Insuasty, B.; Cobo, J.; Nogueras, M. Tetrahedron 2004, 60, 8839. (b) Katritzky, A. R.; Rachwal, B.; Rachwal, S. J. Org. Chem. 1995, 60, 3993. (c) 
Katritzky, A. R.; Abonia, R.; Yang, B.; Qi, M.; Insuasty, B. Synthesis 1998, 1487. (d) Abonia, R.; Albornoz, A.; Insuasty, B.; Quiroga, J.; Meier, H.; Hormaza, A.; Nogueras, M.; Sánchez, A.; Cobo, J.; Low, J. N. Tetrahedron 2001, 57, 4933.

5. (a) Triggle, D. J.; Langs, D. A.; Janis, R. A. Med. Res. Rev. 1989, 9, 123. (b) Triggle, D. J. In Comprehensive Medicinal Chemistry; Emmet, J. C., Ed.; Pergamon: Oxford, 1990; Vol. 3, Chapter 14.1.

6. For review see: (a) Murakami, Y.; Kikuchi, J.-I.; Hisaeda, Y.; Hayashida, O. Chem. Rev. 1996, 96, 721. (b) Stout, D. M.; Meyers, A. I., Chem. Rev. 1982, 82, 223.

7. (a) Yang, J. W.; Hechavarria Fonseca, M. T.; List, B. Angew. Chem. Int. Ed. 2004, 43, 6660. (b) Garden, S. J.; Guìmarães, C. R. W.; Corréa, M. B.; Oliveira, C. A. F. A.; Pinto, C.; Alencastro, R. B. J. Org. Chem. 2003, 68, 8815. (c) Adolfsson, H. Angew. Chem. Int. Ed. 2005, 44, 3340. (d) Zhu, X.-Q.; Liu, Y.-C.; Cheng, J.-P. J. Org. Chem. 1999, 64, 8980.

8. (a) Zehani, S.; Lin, J.; Gelbard, G. Tetrahedron 1989, 45, 733. (b) Burgess, V. A.; Davies, S. G.; Skerlj, R. T.; Whittaker, M. Tetrahedron: Asymmetry 1992, 3, 871. (c) Kanomata, N.; Nakata, T. Angew. Chem., Int. Ed. Engl. 1997, 36, 1207. (d) Obika, S.; Nishiyama, T.; Tatematsu, S.; Miyashita, K.; Iwata, C.; Imanishi, T. Tetrahedron 1997, 53, 593. (e) Rueping, M.; Sugiono, E.; Azap, C.; Theissmann, T.; Bolte, M. Org. Lett. 2005, 7, 3781.

9. (a) Itoh, T.; Nagata, A.; Kurihara, A.; Miyazaki, M.; Ohsawa, A. Tetrahedron Lett. 2002, 43, 3105. (b) Nagata, K.; Itoh, T.; Okada, M.; Ohsawa, A. Tetrahedron 1996, 52, 6569. (c) Itoh, T.; Nagata, K.; Matsuya, Y.; Miyazaki, M.; Ohsawa, A. J. Org. Chem. 1997, 62, 3582.

10. Zhang, J.; Jin, M. Z.; Zhang, W.; Yang, L.; Liu, Z. L. Tetrahedron Lett. 2002, 43, 9687.

11. Robinson, J. M.; Brent, L. W.; Chau, C.; Floyd, K. A.; Gillham, S. L.; McMahan, T. L.; Magda, D. J.; Motycka, T. J.; Pack, M. J.; Roberts, A. L.; Seally, L. A.; Simpson, S. L.; Smith, R. R.; Zalesny, K. N. J. Org. Chem. 1992, 57, 7352.

12. Chebanov, V. A.; Sakhno, Y.; Desenko, S. M.; Chernenko, V. N.; Musatov, V. I.; Musatov, V. I.; Shishkina, S. V.; Shishkin, O. V.; Kappe, C. O. Tetrahedron 2007, 63, 1229.

13. (a) Tomlin, C. D. S., Ed. The Pesticide Manual, 12th ed.; British Crop Protection Council: Farnham, U.K., 2000; pp 413. (b) Caboni, P.; Sammelson, R. E.; Casida, J. E. J. Agric. Food Chem. 2003, 51, 7055 .

14. (a) Skraup, Z. H. Ber. Dtsch. Chem. Ges. 1880, 13, 2086. (b) Fujiwara, H.; Kitagawa, K. Heterocycles 2000, 53, 409. (c) Theoclitou, M.-E.; Robinson, L. A. Tetrahedron Lett. 2002, 43, 3907.

15. Liu, C.; Qian, X.; Sun, G.; Zhao, L.; Li, Z. New J. Chem. 2008, 32, 472. 